\title{
Efectos crónicos de un programa de alargamiento pasivo sobre los valores de la presión arterial sanguínea y el estrés en adultos con hipertensión arterial.
}

\section{Chronics effects of a passive stretching program on the blood pressures and stress levels of hypertensions adults.}

\author{
Alessandro Carielo de Albuquerque \\ Daniel Teixeira Belloni \\ Laboratorio de Biociências da Motricidade Humana - LABIMH-RJ \\ Jorge Isaac Acosta González \\ Director del Departamento de Ciencias del Deporte - FCS/UC - Asunción-Paraguay \\ Elirez Bezerra da Silva \\ Coordenador do Curso de Fisioterapia da Universidade Gama Filho - UGF-Brasil \\ Sílvia Bacelar \\ Laboratório de Biociências da Motricidade Humana - LABIMH-RJ \\ Estélio Henrique Martin Dantas \\ Universidade do Grande Rio - UNIGRANRIO-RJ
}

\section{Resumen}

El objetivo de este estudio fue analizar los efectos crónicos de un programa de alargamiento pasivo sobre la presión arterial sanguínea y estrés en adultos con hipertensión arterial. Participaron de este estudio, 12 personas de ambos sexos, con edad media de $52,12 \pm 5,5$ años, sedentarios y con diagnóstico médico de hipertensión arterial. Las variables recolectadas para la realización de la investigación fueron la presión arterial sanguínea y el estrés de dichas personas. El tratamiento estadístico fue realizado por análisis descriptivos y análisis de inferencia. Los tests estadísticos de naturaleza comparativa fueron realizado a través de los análisis de variancia (ANOVA one-way) yuxtapuesto con el test de Post de variancia (ANOVA one-way) yuxtapuesto con el test de Post
hoc de Tukey, para identificar las posibles diferencias entre los hoc de Tukey, para identificar las posibles diferencias entre los
datos de la presión arterial sanguínea. El test de Kruskal-Wallis datos de la presión arterial sanguínea. El test de Kruskal-Wallis fue usado para comparar las respuestas del cuestionario de
estrés. Fue admitido el valor de $\mathrm{p}<0,05$ como significativo para todos los análisis estadísticos utilizados. Los resultados revelaron que, despues de ocho semanas de tratamiento, los individuos presentaron mejoras significativas $(p<0,05)$ en la presión arterial sanguínea sistólica $(\%=-15,39 ? 13,79 ; p=0,01<0,05)$ y diastólica $(" \%=-14,21 \geq 12,98 ; p=0,00<0,05)$. Con relación al estrés durante el protocolo de alargamiento usado, los sujetos demostraran reducción en el número de individuos que se encontraban tra la fase exhaustiva. Sin embargo se obtuvo un aumento sivo en el numero de individuos clasificados en la fase de resissivo en el número de individuos clasificados en la fase de resisque los ejercicios de alargamiento pasivo presentaron efectos significativos y positivos en la reducción de la presión arterial sanguínea y en la fase del estrés, en as personas con hipertensión arterial, después de ocho semanas de tratamiento.

\section{Abstract}

Purpose of this study was verifier the chronics effects of passive stretching programs on the stress and blood pressure levels of adults with arterial hypertension. 12 subjects of both gender, age average of 52,12 $52,12 \pm 5,52$ year, sedentary and with screening of arterial hypertension, volunteered to participate this study. The variables identified to the conduction of the respective study were the blood pressure, physical activity level and stress of the subjects. Statistic treatment was compost for descriptive and inferential analysis across of the variance analysis test (ANOVA two-way), concomitant with Post hoc test of Tukey, to estimate the differences in the average of the variables during the diverse moments of test. Kuskal-Wallis test was realized to compare the replies of the stress questionnaire. A value of $\mathrm{p}<0,05$ was considered to indicate statistical significance. Results show that after eight week in treatment, subjects demonstrate significant improves $(P<0.05)$ in the systolic blood Pressure ( $\%=-15,39 ? 13,79$; $\mathrm{p}=0,01<0,05)$ y diastolic blood pressure $(\%=$ $14,21212,98 ; p=0,00<0,05)$. Stress levels of the subjects reduced of the exhaustion phase to the resistance phase. In accord to evidences findings, ours conclude that a passive stretching exercise program has positives and significant effects in the blood pressure and stress phase decrease after eight week in treatment.

Palabras clave: presión arterial sanguínea,estrés, alargamiento pasivo, adultos con la enfermedad de hipertensión arterial.

Key words:blood pressure, stress, passive stretching, adults with arterial hypertension disease.

Correspondencia/correspondence: Alessandro Carielo de Albuquerque

Rua Arlete C. Ayres Wanderley, no 336 Sulacap - RJ - CEP 21745-650 Tel (21) 81579972.

E-mail: anatomy.carielo@ig.com.br 


\section{Introducción}

T a hipertensión arterial es una enfermedad que resulta de anomalías en el mecanismo —de control de la presión sanguínea (Ketelhut et al., 1997). La hipertensión arterial era definida como presión arterial sanguínea sistólica, si era mayor que $140 \mathrm{mmHg}$ y presión arterial sanguínea diastólica, si era mayor que 90 mmHg (Palmieri, 2001). Sin embargo, Brandão et al. (2003), actualmente sugieren clasificaciones más rigurosas, proponiendo valores inferiores, como, por ejemplo, hipertensión arterial leve ( $>130$ x 90 mmHg), hipertensión arterial moderada (>160 x 100 mmHg) e hipertensión arterial severa (>180 x $110 \mathrm{mmHg}$ ) dividida por períodos que varían de 1 a 3 . Los autores citados sugieren aún, otra clasificación denominada hipertensión sistólica (>140; >90 $\mathrm{mmHg})$.

Datos estadísticos revelan que aproximadamente 14 millones de brasileños tienen la enfermedad de la hipertensión arterial. De esta enorme cuantidad, adultos en edad económicamente activa ocupan cerca de $15 \%$ de dicho total (King et al. 2004). Consecuentemente, una gran erogación se ocasiona al gobierno del Brasil, principalmente, por el aumento considerable de los costos sociales por invalidez y ausentismo al trabajo (King et al., 2004). Vaccarino (2001) habla también que, 21\% de las jubilaciones son concedidas por diagnóstico médico de enfermedades del sistema circulatorio. De este gran total, 18,1\% tiene como causa de la jubilación el diagnóstico de hipertensión arterial.

Muchos factores se relacionan con la etiología de la enfermedad de la hipertensión arterial, como, por ejemplo, la obesidad, la edad, el tabaquismo, el diabetes mellitus, el sedentarismo y, especialmente, el estrés (Reif et al., 2000). Según Reif et al. (2000), una cierta cuantidad de estrés es normal para ayudar el individuo la superar los desafíos de la vida. Mientras que las condiciones de estrés elevado causan innumerables reacciones desagradables al hombre, llevando a la pérdida de la homeostase orgánica (Losys, 2006).Consecuentemente, tal desequilibrio orgánico ayuda a la aparición de innumerables enfermedades.

El tratamiento de la hipertensión arterial puede ser realizado en forma medicamentosa y no-medicamentosa. Entre las formas de terapia no-medicamentosa, cuyo objetivo primordial es reducir los valores de la presión arterial sanguínea, se destacan la actividad física, la disminución del peso, la restricción dietética de sodio, las técnicas de biofeedback y el relajamiento muscular (Galvão y Junior, 2002; Yung et al. 2001).

Pinto et al. (2003) citan que los ejercicios de alargamiento tienen efectos positivos en pacientes con la enfermedad de la hipertensión arterial. Mientras, Galvão y Junior (2002), relatan que estos ejercicios evitan o eliminan la pérdida de la flexibilidad muscular, reduce el peligro de algunos tipos de lesión, conserva la flexibilidad, excluye o restringe la incomodidad de los nódulos musculares y mejora la circulación sanguínea. 
Estudios actuales han demostrado los efectos positivos de los ejercicios de alargamiento sobre varios componentes de la conducta motora humana. Sin embargo, pocos conocen acerca de las reales repercusiones de este tipo de ejercicio sobre el sistema circulatorio de las personas que tienen la enfermedad de la hipertensión arterial.

La enfermedad de la hipertensión arterial es considerada un grave problema de salud pública en el Brasil, no solamente por la elevada prevalencia de casos, sino principalmente, por la acentuada parcela no diagnosticada o no tratada adecuadamente (Vilacorta ,1999). Debido a que la referida enfermedad acarrea muchas influencias desfavorables al organismo humano, esta problemática ocasiona una preocupación, especialmente por la citada parcela de la población. Por todo ello, el objetivo de este estudio fue verificar los efectos crónicos de un programa de alargamiento pasivo sobre la presión arterial sanguínea y estrés en adultos con la enfermedad de la hipertensión arterial.

\section{Material y métodos}

\section{Muestras}

Los datos de este estudio fueron recolectados de sujetos sedentarios, de ambos sexos, controlados con medicamentos y con diagnóstico médico de hipertensión arterial. Subsiguientemente a los criterios de inclusiónlexclusión, la muestra fue integrada por 12 individuos adultos (media de la edad $=52,12 \pm 5,5$ años) con la enfermedad de la hipertensión arterial (media de la presión arterial sanguínea $=157,16 \pm 24,08 \mathrm{X}$ $95,33 \pm 15,33 \mathrm{mmHg}$ ) y con clasificación de estrés de casi exhaustivo. Todos los testes realizados estaban de acuerdo con las normas para la realización de investigaciones, conforme la orientación del Conselho Nacional de Saúde, respetando las Diretrizes e Normas Regulamentadoras de Pesquisa con seres humanos, vigente desde 10 de octubre de 1996, Resolución numero 251. El presente estudio fue aprobado por el Comité de Ética en Pesquisa de la Universidade Castelo BrancolUCB-RJ.

\section{Procedimientos Metodológicos}

Todos los individuos fueran designados para un sistemático análisis de la fase de estrés en que se encontraban y para la determinación de la presión arterial sanguínea en los momentos iniciales (primer día del tratamiento), durante el tratamiento (último día de la cuarta semana del tratamiento) y después del tratamiento (último día de la octava semana de tratamiento).

La medición de la presión arterial sanguínea fue realizada por método indirecto, utilizando un aparato esfigmomanómetro aneroide de la marca Tycos (USA, 2000) y un estetoscopio de la marca Littemann Quality (Germania, 2003).

Para la realización de la recolección de datos, fueran utilizados tres diferentes protocolos. El primer protocolo, desarrollado por Pierin y Junior (2000), se encargaba 
evaluar la presión arterial sanguínea de los individuos. Posteriormente, el protocolo de Matsudo (2001) fue usado para verificar la condición física que se encontraba cada un de los sujetos. Finalmente, para evaluar el estrés de cada uno de los individuos, fue utilizado el protocolo de Lipp (2000).

El cuestionario usado para la evaluación del estrés, intitulado "Inventario de Síntomas de Estreses para Adultos" (Lipp, 2000), contiene una serie de preguntas divididas en tres cuestiones, subdivididas de la siguiente manera. La cuestión 1 (A y B), se refiere a los síntomas que los sujetos experimentaron en las últimas 24 horas. La cuestión 2 (A y B), es relativa a los síntomas de la última semana. Finalmente, la cuestión 3 (A y B), describen los síntomas del último mes relatados por las personas.

El programa de alargamiento pasivo aplicado en las personas estaba de acuerdo con el protocolo preconizado por Dantas (2005). Este tipo de alargamiento protocolizado, equivale a un alargamiento extenso y completo, realizado para el mantenimiento de la postura corporal, sin sobrepasar el límite máximo del movimiento, durante un tiempo de cuatro para seis segundos. Este programa de alargamiento fue realizado durante ocho semanas, con una frecuencia de tres veces por semana. Las sesiones se realizaron en el horario de las ocho horas, con una repetición por serie y siendo realizadas en forma individual, haciendo que el paciente visualizara el movimiento realizado por el terapeuta y luego ejecutaba los movimientos, en forma totalmente similar. Todas las articulaciones de gran amplitud articular fueran utilizadas, como los de la región del cuello, las articulaciones del hombro, del codo, de la muñeca, de la columna y de las piernas.

\section{Tratamiento Estadístico}

El tratamiento estadístico fue realizado por análisis de naturaleza descriptiva y de inferencia. En la modalidad descriptiva, las medias y los desvíos-padrón de las medias fueron los testes de importancia para los análisis. En los análisis de naturaleza comparativa, fue usado el análisis de variancia (ANOVA one-way) y el teste de Kruskal-Wallis como únicos modelos. Consecuentemente, una ANOVA one-way, yuxtapuesto con el teste de Post Hoc de Tukey, fueron usados para verificar las diferencias determinadas entre la presión arterial sanguínea durante los tres momentos del teste. El teste de Kruskal-Wallis fue hecho para comparar las respuestas del sujeto para las preguntas del cuestionario del estrés durante los variables momentos de la investigación. Fue admitido el valor de p $<0,05$ como significativo para todos los análisis estadísticos utilizados. 


\section{Resultados}

Los resultados de la variación porcentual de la presión arterial sistólica y la presión arterial diastólica están expuestos en el gráfico 1.

Gráfico 1: Variación Porcentual de la Presión Arterial Sanguínea Sistólica y Diastólica
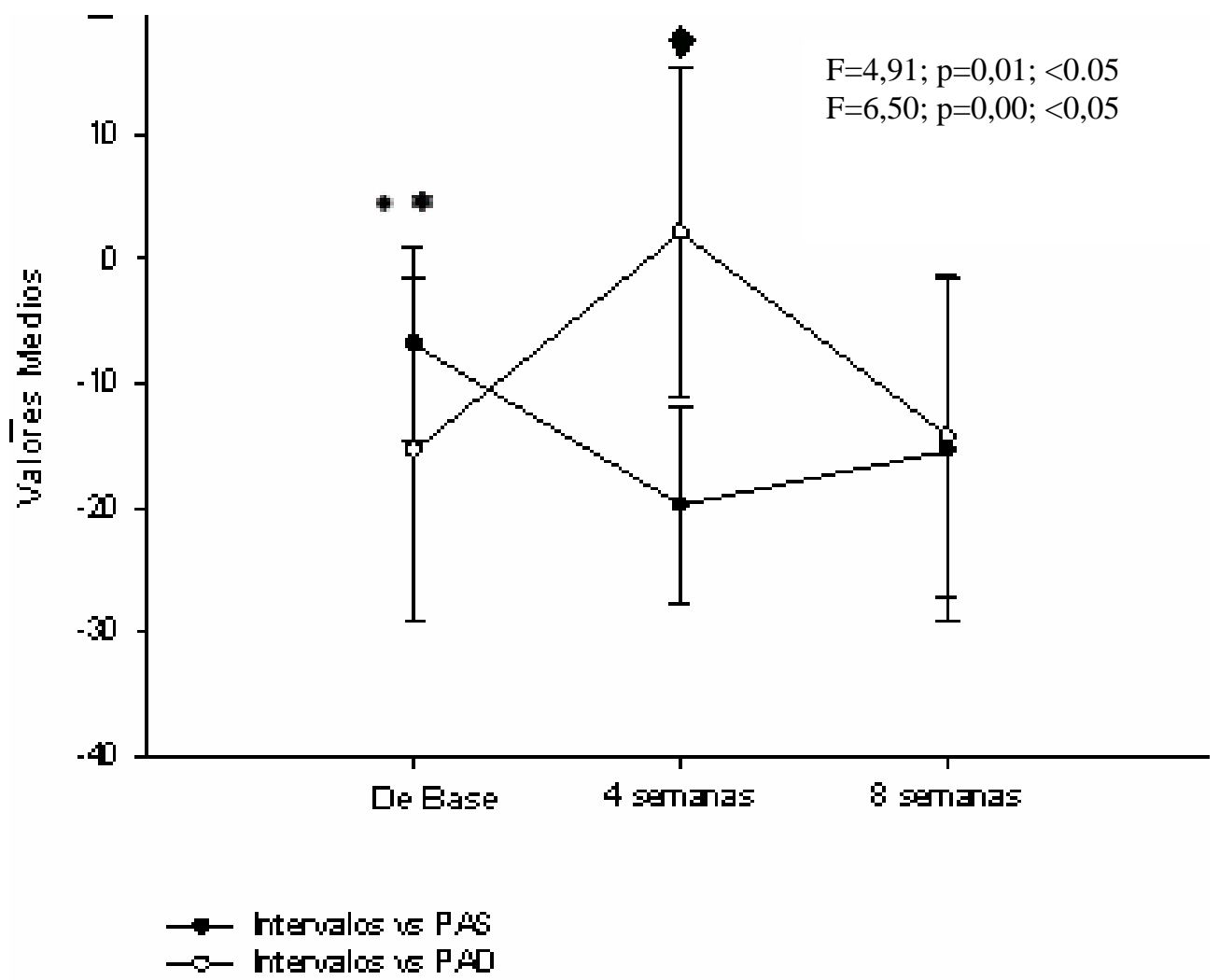

- = Diferencia significativa entre los valores de la presión arterial sanguínea sistólica en el momento inicial y después de cuatro semanas de tratamiento;

* = Diferencia significativa entre los valores de presión arterial sanguínea diastólica en el momento inicial y después cuatro semanas de tratamiento;

- = diferencia significativa entre los valores de presión arterial sanguínea diastólica después cuatro y ocho semanas de tratamiento;

De acuerdo con los datos revelados, se puede observar una reducción $(\mathrm{p}<0,05)$ de los valores de la presión arterial sanguínea sistólica, cuando se comparan al valores de base $(-6,85 \pm 7,84)$, después cuatro $(-19,76 \pm 7,99)$ y ocho $(-15,39 \pm 13,79)$ semanas de tratamiento. Sin embargo, el teste de Post Hoc reveló diferencias estadísticamente significativas solamente entre los valores de base y después de cuatro semanas. Sin embargo, al analizar la presión arterial sanguínea diastólica, las estadísticas revelaran reducciones significativas $(\mathrm{p}<0,05)$ entre todos los valores desarrollados $(-15,39 \pm 13,79)$, después de cuatro semanas $(2,14 \pm 13,17)$ y ocho semanas $(-14,21 \pm 12,98)$, y aún, entre los intervalos de cuatro y ocho semanas $(\mathrm{p}<0,05)$. 
En gráfico 2 están expuestas las clasificaciones de la presión arterial sanguínea. De acuerdo con los resultados, se observa que antes del tratamiento, la mayor parte del grupo de voluntarios fueran clasificados en la etapa de hipertensión grave de la presión arterial sanguínea. Después de cuatro semanas de tratamiento, se constató que los individuos obtuvieron la clasificación normal elevada de la presión arterial sanguínea. Al final del tratamiento, la mayoría de los sujetos obtuvieron la clasificación de la presión arterial sanguínea muy buena.

\section{Gráfico 2: Clasificación de la Presión Arterial Sanguínea}

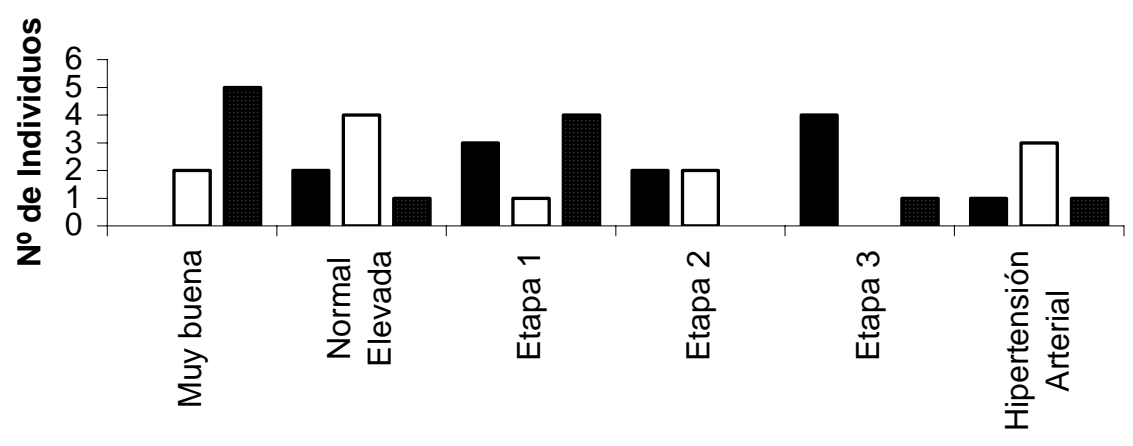

Fase Pre $\square$ Fase Durante $\mathbf{E}$ Fase Pos

Con relación la clasificación del estrés, los resultados se encuentran expuestos en el gráfico 3. En el inicio del tratamiento, los sujetos presentaron la clasificación de exhaustivos y casi exhaustivos. Durante el tratamiento, la clasificación de casi exhaustivos y resistencia se presentaron con mayor frecuencia. Después de ocho semanas de tratamiento, la clasificación resistencia fue la que obtuvo mayor prevalencia entre los valores de clasificación del estrés. Es importante destacar que en este período sólo un individuo se encontraba en la fase exhaustiva.

\section{Grafico 3: Fases del Estrese}

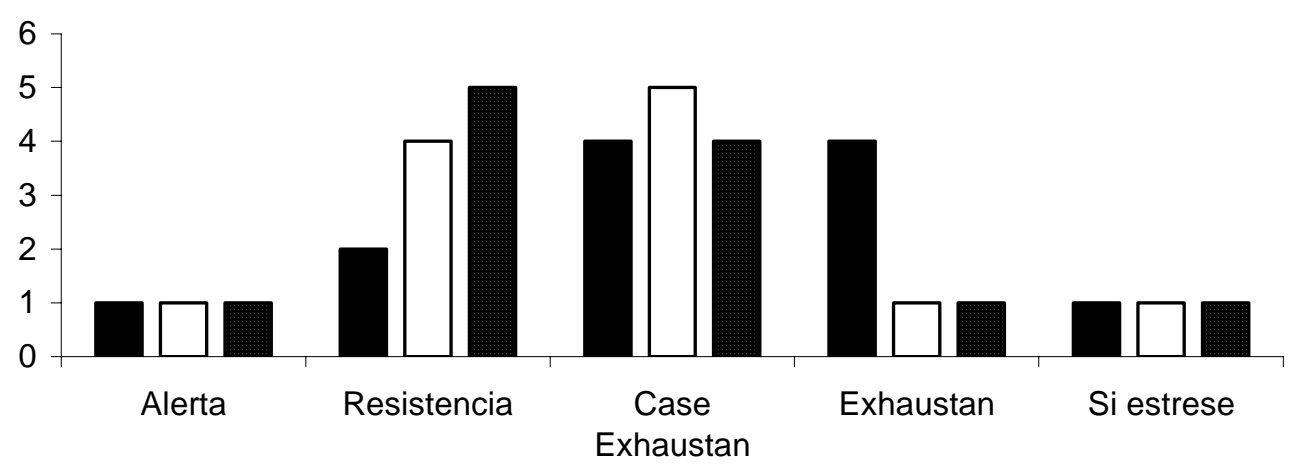

Gase Pre $\square$ Fase Durante $\square$ Fase Pos 
En la tabla 1, se encuentran expuestos los resultados relativos a los síntomas que los individuos experimentaron en las últimas 24 horas, referentes las cuestiones $1 \mathrm{~A}$ y $1 \mathrm{~B}$ del cuestionario de estrés. En la tabla, se observa en la cuestión $1^{\text {a }}$ del cuestionario de estrés, que los ítems que presentaron alteraciones estadísticamente significativa $(\mathrm{p}<0,05)$, fueron la hiperventilación, el cambio del apetito, y también, la taquicardia. En los ítems de las cuestiones 1B, 2 (A y B) y 3 (A y B) ninguna diferenta fue significativa.

Tabla 1: Resultados del Test de Kuskall - Wallis

\begin{tabular}{|l|c|c|c|c|c|c|c|}
\hline \multirow{2}{*}{ Ítems de la Cuestion 1 A } & \multicolumn{3}{|c|}{ Md } & \multicolumn{3}{c|}{ Fr\% } & p-valor \\
\cline { 2 - 9 } & Pre & Durante & Después & Pre & Durante & Después & \\
\hline Taquicardia & 2,00 & 1,00 & 1,00 & 75,00 & 66,70 & 66,70 & 0,06 \\
\hline Hiperventilación & 2,00 & 2,00 & 1,00 & 83,30 & 66,70 & 66,70 & 0,04 \\
\hline Cambio de apetito & 1,00 & 1,00 & 1,00 & 75,00 & 100 & 100 & 0,04 \\
\hline
\end{tabular}

Md = mediana; Fr \% = frecuencia porcentual

\section{Discusión}

Como expresamos anteriormente, diversos factores están correlacionados a la etiología de la hipertensión arterial, como, por ejemplo, la obesidad, la edad, el tabaquismo, la diabetes mellitus, el trabajo, el sedentarismo y el estrés (Reif, 2000). Es bien sabido que una determinada cuantidad de estrés es normal para ayudar el individuo para enfrentar los desafíos de la vida. Sin embargo, índices elevados de estrés causan innumerables reacciones desagradables al organismo del hombre, llevando el estado de estrés elevado a la pérdida de la organic homeostasis (Reif, 2000). Este estudio buscó, a través de una concepción científica, investigar detalladamente los efectos crónicos de un programa de alargamiento pasivo sobre los valores de la presión arterial sanguínea y estrés en adultos que tiene la hipertensión arterial.

La literatura es muy escasa en relación al efecto del alargamiento sobre la hipertensión arterial y el estrés. De todos los estudios disponibles encontrados por los autores, solamente uno informa sobre los efectos positivos del alargamiento sobre la reducción de la presión arterial sanguínea de individuos que tienen hipertensión arterial. Sin embargo, el mismo no ofrece ningún dato cuantitativo como el número de individuos estudiados y los resultados de los valores de presión arterial sanguínea (Pinto et al., 2003).

En presente estudio, verificó que siguiendo el protocolo de alargamiento pasivo se redujo significativamente los valores de presión arterial sistólica $(\Delta \%=-15,39 \pm 13,79$; $\mathrm{p}=0,01<0,05)$ y diastólica $(\Delta \%=-14,21 \pm 12,98 ; \mathrm{p}=0,00<0,05)$, y también, los valores del estrés después ocho semanas de tratamiento.

De acuerdo con Lipp (2000), el estrés puede ser clasificado en fases que varían entre el periodo de alerta (fase positiva del estrés); el periodo de resistencia (fase del alerta 
prolongado, en el que el organismo entra en acción para frenar el desgaste total de energía); fase casi exhaustiva, que puede inducir a enfermedades graves, como hipertensión arterial, y finalmente, el periodo de agotamiento (cuando la tensión ultrapasa el límite del organismo, produciendo mucha ansiedad y efecto negativo sobre el sistema inmunológico). Losyk (2006) al someter a 2300 hombres a diversos factores estresantes, mostró que $72 \%$ presentaron un aumento significativo de la presión arterial, llevando a estas personas al riesgo de tener un accidente cerebral vascular hemorrágico. Cuando esas constataciones son comparadas con los resultados de la presente investigación, las respuestas encontradas comprobaron una relación de dependencia entre las variables.

Otro estudio relata que el estrés puede ser causado por distintos factores, como, por ejemplo, la tensión física, la tensión emocional, la ansiedad, el miedo, la aflicción y la depresión, cuyas las respuestas pueden tener un efecto catastrófico sobre el organismo, incluyendo el sistema cardiovascular, predisponiendo el individuo a la hipertensión arterial (Shuman y Hirst, 1998). En el presente estudio en discusión, los individuos también respondieron que tienen, problemas con la tensión muscular, sensación de desgaste físico, cansancio excesivo, angustia/ansiedad diaria, necesidad de esconderse de todos, pensamiento fijo en una sola cuestión, boca seca e imposibilidad de trabajar, totalmente similar con los datos constatados por Shuman y Hirsh (1998). Jacobson (1993) resalta que los efectos del estrés en el organismo difieren entre los individuos. Consecuentemente, exponen que una variedad de situaciones estresantes en muchos individuos puede generar respuestas cardiovasculares diferentes entre cada uno. Los datos de Jacobson revelaron que algunos pacientes relataban dolor de cabeza, mientras otros sufrían de problemas gástricos o del intestino, bien asma y elevada presión arterial sanguínea. Los datos constatados en nuestro estudio presentaron esa variabilidad de síntomas entre los sujetos, los cuales relataron sentir malestar, náusea, problemas de hiperventilación y elevada presión arterial sanguínea.

El sedentarismo tiene una relación directa con los aumentos de la presión arterial sanguínea. Diversos estudios actuales mencionan que esa conducta de comportamiento es un importante factor de riesgo para enfermedades del sistema cardiovascular (Rique et al. 2002). Segundo Puppin (2003), un estilo de vida sedentario está fuertemente asociado al aumento de la mortalidad y de las enfermedades de las coronarias, así como mayor riesgo de tener hipertensión arterial. En el grupo de personas del estudio actual, todos los individuos eran sedentarios, lo que pudo haber contribuido para los elevados valores de la presión arterial sanguínea encontrados durante el periodo anterior al tratamiento.

Es imprescindible indicar terapias anti-hipertensivas con medicamentos, en situaciones, en los cuales los valores de la presión arterial sanguínea no se modifican por medio del cambio del estilo de vida y después de seis meses de tratamiento no-medicamentoso, así como también en situaciones rígidas, como, por ejemplo, cuando se presentan valores extremamente elevados de la presión arterial sanguínea. Considerando entonces, la recomendación Blumenthal et al. (2002), es importante rehacer el estudio sin que los individuos sujetos hagan uso de medicamento, para que así se pueda confirmar los efectos positivos prevenientes exclusivamente del tratamiento preescrito por los autores. 
De acuerdo con los resultados del estudio en cuestión, se espera que los datos respondan a la duda presentada sobre los efectos crónicos de un programa de alargamiento pasivo sobre los valores de la presión arterial sanguínea y el estrés de adultos con hipertensión arterial.

Conforme a lo expuesto, se recomienda la intervención inmediata de los individuos sedentarios, con presión arterial sanguínea elevada, con altos valores de estrés, al tratamiento más adecuado, y también una detallada investigación de aquel que presenta los síntomas de hiperventilación, cambio de apetito y taquicardia.

A través de los datos detectados, podemos concluir que, el sedentarismo, la hipertensión arterial y el estrés se hallan correlacionados. De este modo, debido a los relevantes beneficios obtenidos en este estudio, podemos considerar al alargamiento pasivo como un importante método no-medicamentoso para el tratamiento de la hipertensión arterial.

Recomendamos la continuidad de ese estudio para verificar los resultados luego de ocho semanas de tratamiento. 


\section{Referencias bibliográficas}

Blumenthal, J.A., Sherwood, A., Gullette, C.D., Georgiades, A., Tweedy, D. (2002). Biobehavioral approaches to the treatment of essential hypertension. Journal of Consulting and Clinical Psychology, 70, 569-589.

Brandão, A.P., Brandão, A.A., Magalhães, M.E.C., Pozzan, R. (2003). Epidemiologia da hipertensão arterial. Revista da Sociedade de Cardiologia do Estado de São Paulo, 13, 7-19.

Dantas, E.H.M. (2005). Alongamento e Flexionamento. 5. ed. Rio de Janeiro: Shape.

Feldman, R.D.(1999). The 1999 Canadian recommendations for the management of hypertension. The Canadian J ournal of Cardiology, 15, 57-64.

Jacob, S.W., Francone, C.A., Lowssow, W.J . (1990). Anatomia e fisiologia humana.. 5. ed. Rio de Janeiro: Guanabara Koogan.

Jacobson, E. (1993). Relax: como vencer as tensões. São Paulo: Cultrix.

Ketelhut, R.G., Franz, I.W., Scholze, J. (1997). Efficacy and position of endurance training as a non-drug therapy in the treatment of arterial hypertension. J ournal of human hypertension, 11, 651-655.

King, D.E., Brent, M.E., Arch, G.M., Mark, E.G. (2004). Elevation of c-reactive protein in people with prehypertension. Mdscape, 10, 37-41.

Lipp, M.N. (2000). Inventário de sintomas de stress para adultos de LIPP. São Paulo: Casa do Psicólogo, 1-5.

Losyk, B. (2006). Estresse \& carreira: o mal e o bem que ele pode fazer a sua vida profissional. São Paulo: Futura.

Matsudo, S. et al. (2001). Questionário internacional de atividade física (IPAQ): estudo de validade e reprodutibilidade no Brasil. Centro de Estudos do Laboratório de Aptidão Física de São Caetano do Sul-CELAFISCS, 6, 1-16.

Palmieri, V. et al. (2001). Relation of various degrees of body mass index in patients with systemic hypertension to left ventricular mass, cardiac output, and peripheral resistance (the hypertension genetic epidemiology network study). The American Journal of Cardiology, 88, 1163-1168.

Pierin, A.M.G., Júnior D.M. (2000). Medida da pressão arterial no paciente obeso: o método indireto com técnica auscultatória e a monitorização ambulatorial. Revista Brasileira de Hipertensão, 7, 161-165.

Puppin, S. (2003). Doenças cardiovasculares: verdades e mitos. Rio de Janeiro: Rio.

Reif, M.H., Field, J., Krasnegorj, J., Theakston, H., Hossain, Z., Burman, I. (2000). High blood pressure and associated symptoms were reduced by massage therapy. Journal of Bodywork and Movement Therapies, 4, 31-38. 
Rique, A.B.R., Soares, E.A., Meirelles, C.M. (2002). Nutrição e exercício na prevenção e controle das doenças cardiovasculares. Revista Brasileira de Medicina do Esporte, 8, 244-252.

Shuman, L.H., Hirsh, H.L. (1998). Stress and health. Trauma, 40, 83-95.

Silva, J.E., Pereira J.S., Natali, A.J., Vale, R.G.S., Dantas, E.H.M. (2006). Efectos crónicos de un programa regular de natación sobre la tensión arterial de adultos hipertensos. Revista Internacional de Ciências Del Deporte, 4, 15-24.

Souza, N.R.M., Silva, N.A.S. (2003). Trabalho e hipertensão arterial. A responsabilidade social das empresas: problemas, oportunidades e possíveis estratégias de intervenção. Revista da SOCERJ , 16, 60-64.

Thomas, J.R., Nelson, J.K. (2002). Métodos de Pesquisa em Atividade Física. 3. ed. Porto Alegre: Artmed.

Vaccarino, V. et al., (2001). Pulse pressure and risk of cardiovascular events in the systolic hypertension in the elderly program. The American Journal of Cardiology, 88, 980-986.

Villacorta, H. (1999). Crise hipertensiva. Revista da SOCERJ, 12, 409-416.

Yung, P., French, P., Leung, B. (2001). Relaxation training as complementary therapy for mild hypertension control and the implications of evidencebasedmedicine. Complementary Therapies in Nursing \& Midwifery, 7, 59-65. 